\title{
Influence of Web Content Management Systems in Web Content Accessibility
}

\author{
Juan Miguel López ${ }^{1}$, Afra Pascual ${ }^{2}$, Llucia Masip ${ }^{2}$, \\ Toni Granollers ${ }^{2}$, and Xavier Cardet ${ }^{2}$ \\ ${ }^{1}$ Department of Computer Languages and Systems, \\ School of Engineering, University of the Basque Country, \\ Nieves Cano 12, 01006 Vitoria-Gasteiz, Spain \\ juanmiguel. lopez@ehu.es \\ ${ }^{2}$ GRIHO research group, Polytechnic School, University of Lleida, \\ Jaume II 69, 25001 Lleida, Spain \\ \{apascual, lluciamaar, tonig\} ediei.udl.cat, \\ xcardet@alumnes.udl.cat
}

\begin{abstract}
Web Content Management Systems (CMS) are traditionally used in institutions to allow web content management by people without technical skills. This study intends to check the influence of the CMS in the accessibility of the contents they handle. First, an accessibility analysis of six widely used CMS is performed base don the Authoring Tool Accessibility Guidelines (ATAG). Second, an accessibility analysis of a series of city council web pages managed by abovementioned CMS by using Web Content Accessibility Guidelines (WCAG). Results of the study show that although web pages managed by the CMS with a better degree of ATAG fulfilment provide better accessibility, there is no direct correlation between results obtained in both evaluations. Information about what aspects cause accessibility errors in the CMS and the impact of such aspects in accessible web content management is also provided.
\end{abstract}

Keywords: Web Content Management Systems, Authoring Tool Accessibility Guidelines, Web Content Accessibility Guidelines.

\section{Introduction}

Currently, there are powerful legal requirements to take accessibility into account as a requirement for developing web sites. For instance, these kinds of regulations have been performed on European Union countries [1]. It is important to note that these legal frameworks do not only affect public administration, but also corporations that develop software or provide services for their use in civil service.

Nowadays there is a wide range of institutions that use CMS to manage their web content. They are especially useful as they allow users without technical skills to introduce eliminate or modify web content, but it does have some risks regarding accessibility [2] [3]. Furthermore, CMS generally do not generate accessible web content by default and allow users to perform few modifications to improve the 
accessibility of the web content they manage. These kinds of modifications are frequently insufficient to allow websites to be accessible for people with disabilities [4].

\section{Study}

Six CMS were selected for the study. The selection was based on the CMS to be open source, commonly used in civil service and general purpose systems. Selected CMS were: Plone, Joomla!, Typo3, EzPublish, OpenCMS and Drupal. First, an accessibility analysis of the CMS on their default installations was performed based on the Authoring Tool Accessibility Guidelines (ATAG) [5]. In order to complete the study, a web content accessibility analysis was performed on a sample of 90 worldwide town council web pages, 15 of them managed by each CMS. Web Content Accessibility Guidelines (WCAG) [6] were used for analysing the accessibility of each web page using the evaluation methodology established by the World Wide Web Consortium [7]. A total of three evaluators performed the study, each of them evaluating two CMS. Although it would have been desirable that every CMS would have been evaluated by more that one evaluator, the complexity of CMS led to estimate positively the fact that evaluators had a high level of knowledge regarding CMS to be evaluated. The results obtained by these evaluators were estimated to be more useful compared to the ones that people without such knowledge should provide. Evaluators also had deep knowledge on both accessibility guidelines used in this study.

Table 1. Quantitative results of the ATAG evaluation for every selected CMS

\begin{tabular}{llllllllllllllllllll}
\hline Priority & \multicolumn{3}{c}{ Drupal } & \multicolumn{1}{c}{ EzPublish } & \multicolumn{1}{c}{ Joomla! } & \multicolumn{1}{c}{ OpenCMS } & \multicolumn{3}{c}{ Plone } & \multicolumn{3}{c}{ Typo3 } \\
\hline & C & Ac & I & C & Ac & I & C & Ac & I & C & Ac & I & C & Ac & I & C & Ac & I \\
1 & 3 & 2 & 3 & 3 & 0 & 5 & 2 & 1 & 5 & 1 & 1 & 6 & 3 & 3 & 2 & 2 & 1 & 5 \\
2 & 1 & 0 & 6 & 2 & 0 & 5 & 0 & 4 & 3 & 1 & 1 & 5 & 1 & 5 & 1 & 1 & 1 & 5 \\
3 & 1 & 0 & 4 & 1 & 0 & 4 & 0 & 2 & 3 & 0 & 0 & 5 & 1 & 3 & 1 & 0 & 1 & 4 \\
Relative & 0 & 0 & 7 & 1 & 0 & 6 & 0 & 2 & 5 & 0 & 0 & 7 & 3 & 1 & 3 & 0 & 2 & 5
\end{tabular}

Table 2. Sum of detected accessibility errors for all web pages of each analysed CMS

\begin{tabular}{ccccccc}
\hline Priority & Drupal & EzPublish & Joomla! & OpenCMS & Plone & Typo3 \\
\hline 1 & 20 & 26 & 32 & 83 & 248 & 39 \\
2 & 341 & 167 & 566 & 1265 & 422 & 571 \\
3 & 83 & 98 & 219 & 168 & 169 & 105
\end{tabular}

Table 3. Mean and standard deviation of detected accessibility errors for all web pages of each analysed CMS

\begin{tabular}{ccccccccccccc}
\hline Priority & \multicolumn{2}{c}{ Drupal } & \multicolumn{2}{c}{ EzPublish } & \multicolumn{2}{c}{ Joomla! } & \multicolumn{2}{c}{ OpenCMS } & \multicolumn{2}{c}{ Plone } & \multicolumn{2}{c}{ Typo3 } \\
\hline & $\mathrm{M}$ & $\mathrm{St}$ & $\mathrm{M}$ & $\mathrm{St}$ & $\mathrm{M}$ & $\mathrm{St}$ & $\mathrm{M}$ & $\mathrm{St}$ & $\mathrm{M}$ & $\mathrm{St}$ & $\mathrm{M}$ & $\mathrm{St}$ \\
1 & 1,3 & 2,4 & 1,7 & 2,7 & 2,1 & 2,6 & 5,5 & 14,5 & 16,5 & 15,5 & 2,6 & 4,5 \\
2 & 22,7 & 32,3 & 11,1 & 14,1 & 37,7 & 44,1 & 84,3 & 98,2 & 27,8 & 30,5 & 38,1 & 70,7 \\
3 & 5,5 & 4,1 & 6,5 & 6,9 & 14,6 & 16,8 & 11,2 & 13,4 & 12 & 12 & 7,0 & 9,4
\end{tabular}


Table 1 summarizes the results obtained in the ATAG evaluation, grouped by CMS. Rows are organized by the type of ATAG priority. Columns are labelled as: "C" (indicates the number of guidelines that were correctly fulfilled), "Ac" (indicates the number of guidelines with almost all requirements satisfied or bugs need to be fixed) or I (indicates the number of guidelines that were not correctly fulfilled). Guidelines with relative priority were evaluates separately, although it must be taken into account that they can be interpreted as different priority depending on the case. Table 2 resumes the results obtained in performed web accessibility evaluations. Due to space constraints, results are expressed by means of the number of accessibility errors for the different analysed guidelines, grouped by priorities. It must be taken into account that the table sums up all errors located for the 15 web pages analysed per CMS. On the other hand, Table 3 shows the mean ("M") and the standard deviation ("St") of the accessibility errors detected for the 15 web pages analysed per CMS, ordered by priority.

\section{Discussion and Conclusions}

Regarding quantitative results corresponding to the six analysed CMS, Drupal and Plone show more complete features regarding accessibility in their default installation. Anyhow, none of them achieved an "A" level of compliance regarding ATAG fulfilment. One of the main issues arising from the study is that the web editors used by default in analysed CMS do not allow generating accessible content. In this sense, they must be reconfigured, be discarded or allow installing web content editors external to the CMS so users can generate accessible web content. Another relevant aspect is the need to change default CMS templates, as they cause web elements' layout not to be accessible. The need to improve documentation regarding the accessibility that CMS provide is another aspect to assess.

Regarding the quantitative analysis of the web content accessibility of the sample of analysed web pages, it is remarkable the fact that a minimum number of web pages reaches even the "A" compliance level established by WCAG 1.0 [6], despite the legality regarding accessibility that analysed web pages should fulfil. Interestingly, it is worth noting that only 1 web page managed by Plone and 9 managed by Drupal achieve such compliance level. Accessibility errors found in these web pages are due to a series of reasons:

1. Most priority 1 errors were due to the lack of a proper alternative text in images (WCAG 1.1 checkpoint)

2. A large number of web pages used absolute measures instead of relative ones to position elements in web pages (WCAG 3.4 checkpoint).

3. Use of device-dependent events (associated only to mouse events by) and lack of redundancy for these cases.

4. The fact that default installation of most CMS (except for Plone and Drupal) did not provide by default a clear content and layout separation has led to accessibility errors in almost all the web paged managed by them (WCAG 3.3 and 11.2 checkpoints).

The results of CMS accessibility evaluation show no direct correlation with the results of web accessibility evaluation. For instance, Plone shows relatively good results 
regarding ATAG fulfilment, but poor results regarding the accessibility of web pages managed using this CMS compared to other CMS. However, web pages managed using Drupal and EzPublish show less accessibility errors compared to the rest.

This work intends to highlight the necessity of the CMS to manage adequately the accessibility of the contents they manage. One interesting conclusion derived from this work is that all analysed CMS can be configured so they can be accessible. In order to do so, it is necessary to modify internal CMS classes and templates that adjust web content in an accessible way. Besides, the use of web content editors external to the CMS can allow managing accessible web code. There is also the option of including plugins in all the CMS that can be used to manage concrete aspects and can be used as tools to improve accessibility. Anyhow, all these solutions require deep and exhaustive knowledge regarding each CMS. There are some resources that allow improving CMS accessibility [8] [9], but they do not provide a clear methodological approach in providing different concrete solutions. Furthermore, it is important to provide accessibility monitoring systems to check the accessibility of managed web content [10].

Acknowledgements. This work has been partially funded by Spanish Ministry of Science and Innovation through TIN2008-06228 and TIN2008-06596-C02-01 research projects. Llucia Masip is the recipient of a pre-doctoral fellowship from the University of Lleida.

\section{References}

1. Article 26 of the European Union Charter of Fundamental Rights (extracted on June 2011 from), http: / / www. europarl.europa.eu/charter/pdf / text_en.pdf

2. Burzagli, L., Billi, M., Gabbanini, F., Graziani, P., Palchetti, E.: The use of current content management systems for accessibility. In: Miesenberger, K., Klaus, J., Zagler, W.L., Burger, D. (eds.) ICCHP 2004. LNCS, vol. 3118, pp. 331-338. Springer, Heidelberg (2004)

3. Rainville-pitt, S., D'Amour, J.M.: Using a CMS to create fully accessible websites. In: W4A2007- ACM International Conference Proceeding Series (2007)

4. Schulz, M., Pieper, M.: Web Compliance Management: Barrier-Free Websites Just by Simply Pressing the Button? Accessibility and the Use of Content-Management-Systems. In: Stephanidis, C., Pieper, M. (eds.) ERCIM Ws UI4ALL 2006. LNCS, vol. 4397, pp. 419-426. Springer, Heidelberg (2007)

5. Treviranus, J., McCathieNevile, C., Jacobs, I., Richards, J.: Authoring Tool Accessibility Guidelines 1.0. (extracted on June 2011 from), http://Www.w3.org/TR/WAIAUTOOLS /

6. Chisolm, W., Vanderheiden, G., Jacobs, I.: Web Content Accessibility Guidelines 1.0. (extracted on June 2011 from), http: / / www . w3 . org/TR/WAI-WEBCONTENT /

7. Evaluating Web Sites for Accessibility: Overview, (extracted on June 2011 from), http: / /www.w3 . org/WAI / eval / Overview.html

8. Connor, J.O. Choosing an Accessible CMS, (extracted on June 2011 from), http: / / juicystudio.com/article/choosing-an-accessible-cms.php

9. NCDAE Tips and Tools: Content Management Systems \& Accessibility, (extracted on June 2011 from), http: / / www. ncdae.org/tools / fact sheets / cms . cfm

10. López, J.M., Pascual, A., Granollers, A.: Engineering Accessibility in Web Content Management System Environments. In: Federrath, H. (ed.) Designing Privacy Enhancing Technologies. LNCS, vol. 2009, pp. 597-604. Springer, Heidelberg (2001) 